\section{RT-induzierten Knochenschmerzen vorbeugen}

\author{
Eine palliative Radiotherapie symptomatischer Knochenmetastasen kann \\ vorübergehend Schmerzen aufflackern lassen. In einer placebokontrollierten \\ Studie wurde getestet, ob Dexamethason dies wirksam verhindert.
}

$\mathrm{N}$ ach palliativer Radiotherapie (RT) verschlechtern sich symptomatische Knochenschmerzen in 30-40\% der Fälle vorübergehend. Vermutlich ist dies durch inflammatorische Zytokine bedingt, weshalb sich Dexamethason zur Prophylaxe eignen könnte. In einer doppelblinden Phase-III-Studie erhielten 298 Patienten randomisiert oral entweder 4 mg Dexamethason $(n=148)$ oder Placebo $(n=150)$ 1 Stunde vor Beginn der Radiotherapie (Einzeldosis von 8 Gy auf eine Knochenmetastase an Tag 0) und an den folgenden 4 Tagen (Tag 1-4). Sie litten am häufigsten an Lungen- (28\%), Prostata- (25\%) oder Mammakarzinomen (22\%). Vor der Therapie und in den 10 Tagen danach wurden sie täglich zu Schmerzen und Opioidbedarf befragt. Zu Beginn sowie an den Tagen 10 und 42 nach Radiothe- rapie füllten sie zudem den EORTC QLQC15-PAL, das Bone Metastases Module (EORTC QLQ-BM22) und den Dexamethasone Symptom Questionnaire aus. Als Schmerzaufflackern galt eine Zunahme der Schmerzen um mindestens 2 Punkte auf einer Skala von $0-10$, ohne dass die Schmerztherapie reduziert worden war, oder eine $25 \%$ ige Dosissteigerung der Schmerztherapie ohne Verbesserung der Schmerzen an den Tagen 0-10, gefolgt von einem Rückgang auf das Ausgangsniveau oder darunter.

39 Patienten der Dexamethason- (26\%) und 53 der Placebogruppe (35\%) erlebten in den Tagen 0-10 ein Schmerzaufflackern $(\mathrm{p}=0,05)$, an den Tagen $0-5$ waren es $29(20 \%)$ versus 46 (31\%) Patienten (p $=0,03$ ). Zudem waren die Schmerzen unter Dexamethason an Tag 10 gegenüber
Tag 0 stärker zurückgegangen als unter Placebo, allerdings nicht signifikant $(\mathrm{p}=$ $0,09)$. Signifikant hingegen war der stärkere Schmerzrückgang an den Tagen 0-5 unter Dexamethason im Vergleich zu Placebo $(p=0,01)$. An Tag 10 hatten die Patienten der Dexamethason-Gruppe deutlich weniger Übelkeit und seltener funktionale Beeinträchtigungen als zu Studienbeginn. Auch im Vergleich zur Placebogruppe ging es ihnen hinichtlich dieser Endpunkte besser, zudem war ihr Appetit besser.

Fazit: Im Vergleich zu Placebo reduziert Dexamethason das Auftreten RT-induzierter Schmerzen bei der Bestrahlung von Knochenmetastasen. Des Weiteren verbessern sich Übelkeit, Appetit und funktionale Beeinträchtigungen ohne schwerwiegende Nebenwirkungen.

Kathrin von Kieseritzky

Chow $\mathrm{E}$ et al. Dexamethasone in the prophylaxis of radiation-induced pain flare after palliative radiotherapy for bone metastases: a double-blind, randomised placebo-controlled, phase 3 trial. Lancet Oncol. 2015; 16(15):1463-72

\title{
Niedrig dosiertes Morphin vs. schwaches Opioid
}

Reicht es aus, unter moderaten Schmerzen leidende onkologische Patienten mit einem schwachen Opioid zu behandeln, oder sollten sie besser niedrig dosiertes Morphin erhalten? Dieser Frage gingen Forscher aus Italien nach.

Hier steht eine Anzeige.

Springer

Moderate Tumorschmerzen sollten laut WHO-Stufenschema mit schwachen Opioiden behandelt werden.
E ine gute Schmerztherapie ist eine der _ zentralen Säulen, um die Lebensqualität von Krebspatienten so lange wie möglich zu erhalten. Der 3-Stufen-Plan der WHO sieht auf Stufe II (leichte bis mittlere Schmerzen) schwache Opioide vor (z. B. Codein, Tramadol), auf Stufe III (starke Schmerzen) starke Opioide. Seit geraumer Zeit mehren sich Hinweise, dass starke Opioide schon bei moderaten Schmerzen vorteilhafter sein könnten.

In einer Phase-III-Studie wurde bei opioidnaiven Patienten mit moderaten Tumorschmerzen eine Therapie mit niedrig dosiertem Morphin oder einem standarddosierten schwachen Opioid verglichen. In der Studie wurden 240 erwachsene Krebspatienten auf das niedrig dosierte Morphin (M-Gruppe; $\mathrm{n}=118$ ) oder ein schwaches Opioid (WO-Gruppe; $\mathrm{n}=122$ ) über 28 Tage randomisiert. Primärer Endpunkt war das Ansprechen, also ein Rückgang der Schmerzintensität um $20 \%$ auf einer numerischen Skala.

88,2\% der M-Gruppe und 57,7\% der WO-Gruppe erreichten dieses Ziel (Odds Risk 6,18; $\mathrm{p}<0,001$ ). Eine klinisch bedeutsame $(\geq 30 \%)$ und hoch bedeutsame Schmerzreduktion ( $\geq 50 \%$ ) im Vergleich zu Studienbeginn wurde signifikant häufiger unter niedrig dosiertem Morphin erreicht $(p<0,001)$. Weil die Schmerztherapie nicht befriedigend war, wechselten 41 Patienten (35\%) der WO-Gruppe auf ein starkes Opioid und 17 Patienten (15,5\%) der M-Gruppe auf ein anderes starkes Opioid ( $\mathrm{p}=$ 0,001). 28,2\% (33/117) der WO- und 13,6\% (15/110) der M-Gruppe benötigten eine Dosissteigerung $(\mathrm{p}=0,007)$. Der Opioid-Eskalations-Index war in der MGruppe niedriger als in der WO-Gruppe $(4,76$ vs. 8,$76 ; \mathrm{p}=0,002)$.

Gemessen am Gesamtsymptomwert im Edmonton Symptom Assessment 